\title{
Non-Forward BFKL at Next-to-Leading Approximation ${ }^{\prime}$
}

\author{
V. Fadin \\ Budker Institute for Nuclear Physics and \\ Novosibirsk State University, 630090 Novosibirsk, Russia
}

\begin{abstract}
Representation of non-forward scattering amplitudes in the BFKL approach is discussed and the results obtained in the next-to leading order are briefly reviewed.
\end{abstract}

\section{Introduction}

The BFKL approach [1] gives the most common basis for the description of processes at large centre-of-mass energy $\sqrt{s}$ and fixed (not growing with $s$ ) momentum transfers $\sqrt{-t_{i}}$ in the framework of perturbative QCD. This approach was developed originally in the leading logarithmic approximation (LLA), which means resummation of all terms of the type $\left[\alpha_{s} \ln s\right]^{n}$ $\left(\alpha_{s}=g^{2} /(4 \pi)\right.$ is the QCD coupling constant). Such approximation leads to a sharp increase of cross sections with $s$ :

$$
\sigma_{\text {tot }}^{L L A} \sim \frac{s^{\omega_{P}^{B}}}{\sqrt{\ln s}}, \quad \omega_{P}^{B}=\frac{g^{2}}{\pi^{2}} N \ln 2,
$$

where $\omega_{P}^{B}$ is the LLA position of the most right singularity in the complex momentum plane of the $t$-channel partial wave with vacuum quantum numbers (Pomeron singularity), $N$ is the number of colours $\left(N=3\right.$ for QCD). The Froissart bound $\sigma_{t o t}<C(\ln s)^{2}$ is violated in LLA, so that LLA can not be applied at asymptotically large energies. The problem of unitarization of LLA results is extremely important from the theoretical point of view. But in the region of energies accessible for modern experiments it seems that the most important disadvantage of LLA is that neither the scale of $s$ nor the argument of the running coupling constant $\alpha_{s}$ are fixed in this approximation. To remove these uncertainties we have to know radiative corrections to LLA.

The calculation of the radiative corrections has taken many years of a hard work. Two years ago the kernel of the BFKL equation was obtained in the next-to-leading order (NLO) [2], 3] for the case of the forward scattering, i.e. for momentum transfer $t=0$ and the singlet colour representations the $t$-channel. In the $\overline{M S}$ renormalization scheme with a reasonable scale setting the corrections appear to be large, although they are not so large in non-Abelian physical renormalization schemes with BLM scale setting [4]. The problem of large corrections was widely discussed in literature (see, for instance [5]). Several ways to cure the problem were suggested which provide an opportunity for application of the BFKL approach at NLO to high energy phenomenology.

An important way of development of the BFKL approach became the generalization of the obtained results to the non-forward scattering [6]. For singlet colour representation in the $t$-channel, the generalized approach can be used directly for the description of a wide circle of physical processes, in particular, diffraction phenomena. The generalization for non-singlet colour states is also of great importance, especially for the antisymmetric colour octet state

\footnotetext{
${ }^{1}$ Work supported in part by INTAS and in part by the Russian Fund of Basic Researches.

${ }^{2}$ The speaker thanks R. Fiore, M. Kotsky and A. Papa, his collaborators in works on which this talk is based.
} 
of two Reggeized gluons in the $t$-channel. This case is especially important since the BFKL approach is based on the gluon Reggeization, although the Reggeization is not proved at NLO. Therefore, at this order the Reggeization is a hypothesis which must be carefully checked. This can be done using the "bootstrap" equations [6] appearing from the requirement of the compatibility of the gluon Reggeization with the $s$-channel unitarity.

In the next Section we remind the representation of scattering amplitudes in the BFKL approach. In Section 3 we discuss the results obtained for the non-forward BFKL kernel and present some of them. In Section 4 the same is done for the impact factors. The problem of "bootstrap" is discussed in Section 5.

\section{Scattering amplitudes in the BFKL approach}

The BFKL approach is based on the remarkable property of QCD - the gluon Reggeization. Remind that we use the notion "Reggeization" in a strong sense [6]. For the process $A+B$ $\rightarrow A^{\prime}+B^{\prime}$ at large $s$ and fixed $t\left(s=\left(p_{A}+p_{B}\right)^{2}, t=q^{2}, q=p_{A}-p_{A^{\prime}}\right)$ it means that the part of the amplitude corresponding to the gluon quantum numbers (colour octet state and negative signature) in the $t$-channel has the Regge form

$$
\left(\mathcal{A}_{8^{-}}\right)_{A B}^{A^{\prime} B^{\prime}}=\Gamma_{A^{\prime} A}^{c}(q)\left[\left(\frac{-s}{-t}\right)^{j(t)}-\left(\frac{s}{-t}\right)^{j(t)}\right] \Gamma_{B^{\prime} B}^{c}(q)
$$

where $c$ is a color index and $j(t)$ is the Regge trajectory passing through zero at $t=1$. Therefore the gluon in QCD lies on the Regge trajectory $j(t)=1+\omega(t)$ with $\omega(0)=0$, so that it appears to be not an elementary object (as well as quark, see [7]). $\Gamma_{P^{\prime} P}^{c}$ in (2) are the particle-particleReggeon (PPR) vertices which do not depend on $s$. Notice that (2) represents correctly the analytical structure of the scattering amplitude, which is quite simple in this case.

The amplitudes with quantum numbers in the $t$ - channel different from the gluon ones are obtained in the BFKL approach using the unitarity relations. For this purpose one needs to know inelastic amplitudes. The main contributions in the unitarity relations come from the multi-Regge kinematics, where all produced particles are strongly ordered in the rapidity space and all their transverse momenta are fixed (not growing with $s$ ). In LLA only such kinematics does contribute. In the next-to-leading approximation (NLA), where terms with one extra power of $\alpha_{s}$ compared to LLA are kept, production of a pair of particles with rapidities of the same order is also admitted.

The production amplitudes have a complicated analytical structure even in the multi-Regge kinematics. Fortunately, we need to know only real parts of these amplitudes. Due to the gluon Reggeization they have a simple factorized form and can be presented as

$\mathcal{A}_{A B}^{\tilde{A} \tilde{B}+n}=2 s \Gamma_{\tilde{A} A}^{c_{1}}\left(q_{1}\right)\left[\prod_{i=1}^{n} \frac{1}{t_{i}} \gamma_{c_{i} c_{i+1}}^{P_{i}}\left(q_{i}, q_{i+1}\right)\left(\frac{s_{i}}{\sqrt{\vec{k}_{i-1}^{2} \vec{k}_{i}^{2}}}\right)^{\omega\left(t_{i}\right)}\right] \frac{1}{t_{n+1}}\left(\frac{s_{n+1}}{\sqrt{\vec{k}_{n}^{2} \vec{k}_{n+1}^{2}}}\right)^{\omega\left(t_{n+1}\right)} \Gamma_{\tilde{B} B}^{c_{n+1}}\left(q_{n+1}\right)$,

where $\gamma_{c_{i} c_{i+1}}^{P_{i}}\left(q_{i}, q_{i+1}\right)$ are the so-called Reggeon-Reggeon-particle (RRP) vertices, i.e. the effective vertices for the production of the particles $P_{i}$ with momenta $k_{i}=q_{i}-q_{i+1}$ in the collision of Reggeons with momenta $q_{i}$ and $-q_{i+1}$ and colour indices $c_{i}$ and $c_{i+1}, q_{0} \equiv p_{A}, q_{n+1} \equiv-p_{B}, s_{i}=$ $\left(k_{i-1}+k_{i}\right)^{2}, k_{0} \equiv p_{\tilde{A}}, k_{n+1} \equiv p_{\tilde{B}}$, vector sign is used for denotation of components of momenta transverse to the plane of the initial momenta $p_{A}, p_{B}$. Pay attention that we have taken definite energy scales in the Regge factors in (3) as well as in (2). In principle, we could take an arbitrary scale $s_{R}$ [8]; in this case the PPR and RRP vertices would become dependent on $s_{R}$. Of course, physical results do not depend on the scale. 
In LLA only one particle can be produced in each RRP vertex, and since our Reggeons are Reggeized gluons, this particle can be only a gluon. The situation changes in NLA, where one of the vertices can represent production of a pair of particles with fixed (not increasing with $s$ ) invariant mass. We can treat this kinematics using the effective vertices $\gamma_{c c^{\prime}}^{G G}$ and $\gamma_{c c^{\prime}}^{Q \bar{Q}}$ for production of two gluons and a quark-antiquark pair in Reggeon-Reggeon collisions. Analogously, we use effective vertices $\Gamma_{\{(f)\} P}^{c}$ for production of states $\{(f)\}$ containing an extra particle in the fragmentation region of the particle $P$ in the process of scattering of this particle off the Reggeon.

In the BFKL approach Pomeron appears as a compound state of two Reggeized gluons in the $t$ - channel in the colour singlet representation. In general case, the amplitude for the high energy process $A+B \rightarrow A^{\prime}+B^{\prime}$ at fixed momentum transfer $\sqrt{-t}$ is presented in this approach as the convolution of the impact factors $\Phi_{A^{\prime} A}$ and $\Phi_{B^{\prime} B}$, which describe the $A \rightarrow A^{\prime}$ and $B \rightarrow B^{\prime}$ transitions in the particle-Reggeon scattering processes, and the Green's function $G$ for Reggeon-Reggeon scattering [6]:

$$
\begin{aligned}
\mathcal{A}_{A B}^{A^{\prime} B^{\prime}}= & \frac{i s}{(2 \pi)^{D-1}} \int \frac{d^{D-2} q_{1}}{\vec{q}_{1}^{2} \vec{q}_{1}^{\prime 2}} \int \frac{d^{D-2} q_{2}}{{\overrightarrow{q_{2}}}_{2}^{2} \vec{q}_{2}^{\prime 2}} \int_{-i \infty+\delta}^{i \infty+\delta} \frac{d \omega}{\sin (\pi \omega)} \sum_{\mathcal{R}, \nu} \Phi_{A^{\prime} A}^{(\mathcal{R}, \nu)}\left(\overrightarrow{q_{1}} ; \vec{q} ; s_{0}\right) \\
& \times\left[\left(\frac{-s}{s_{0}}\right)^{\omega}-\tau\left(\frac{s}{s_{0}}\right)^{\omega}\right] G_{\omega}^{(\mathcal{R})}\left(\vec{q}_{1}, \overrightarrow{q_{2}}, \vec{q}\right) \Phi_{B^{\prime} B}^{(\mathcal{R}, \nu)}\left(-\vec{q}_{2} ;-\vec{q} ; s_{0}\right) .
\end{aligned}
$$

Here and below $q_{i}^{\prime} \equiv q_{i}-q, \overrightarrow{q_{i}}$ are $D-2$ dimensional vectors $(D=4+2 \epsilon$ is the space-time dimension different from 4 to regularize the infrared singularities), $q \simeq \vec{q}$ is the momentum transfer in the process $A+B \rightarrow A^{\prime}+B^{\prime}$. The sum in (国) is taken over irreducible representations $\mathcal{R}$ of the colour group, which are contained in the product of two adjoint representations, and over states $\nu$ from full sets of states for these representations; $\tau$ is the signature equal $+1(-1)$ for symmetric (antisymmetric) representations and $s_{0}$ is an energy scale. $\Phi_{A^{\prime} A}^{(\mathcal{R}, \nu)}$ and $\Phi_{B^{\prime} B}^{(\mathcal{R}, \nu)}$ are the projected on the states $(\mathcal{R}, \nu)$ impact factors, and $G_{\omega}^{(\mathcal{R})}$ are the Mellin transforms of the Green's functions for Reggeon-Reggeon scattering. All dependence from $s$ is determined by $G_{\omega}^{(\mathcal{R})}$, which obey the equation [6]:

$$
\omega G_{\omega}^{(\mathcal{R})}\left(\vec{q}_{1}, \vec{q}_{2}, \vec{q}\right)=\vec{q}_{1}^{2} \vec{q}_{1}^{\prime 2} \delta^{(D-2)}\left(\vec{q}_{1}-\vec{q}_{2}\right)+\int \frac{d^{D-2} r}{\vec{r}^{2} \vec{r}^{\prime 2}} \mathcal{K}^{(\mathcal{R})}\left(\overrightarrow{q_{1}}, \vec{r} ; \vec{q}\right) G_{\omega}^{(\mathcal{R})}\left(\vec{r}, \overrightarrow{q_{2}} ; \vec{q}\right)
$$

where the kernel is given by the sum of the "virtual" part, defined by the gluon trajectory $j(t)=1+\omega(t)$, and the "real" part $\mathcal{K}_{r}^{(\mathcal{R})}$, related to the real particle production in ReggeonReggeon collisions:

$$
\mathcal{K}^{(\mathcal{R})}\left(\vec{q}_{1}, \vec{q}_{2} ; \vec{q}\right)=\left[\omega\left(-\vec{q}_{1}^{2}\right)+\omega\left(-\vec{q}_{1}^{\prime 2}\right)\right] \vec{q}_{1}^{2} \vec{q}_{1}^{\prime 2} \delta^{(D-2)}\left(\vec{q}_{1}-\vec{q}_{2}\right)+\mathcal{K}_{r}^{(\mathcal{R})}\left(\vec{q}_{1}, \overrightarrow{q_{2}} ; \vec{q}\right) .
$$

In LLA both the gluon trajectory and the "real" part of the kernel are pure gluonic:

$$
\omega^{(1)}(t)=\frac{g^{2} N t}{2(2 \pi)^{D-1}} \int \frac{d^{D-2} q_{1}}{\vec{q}_{1}^{2} \vec{q}_{1}^{\prime 2}}=-\frac{g^{2} N \Gamma(1-\epsilon) \Gamma^{2}(\epsilon)}{(4 \pi)^{D / 2} \Gamma(2 \epsilon)}\left(\vec{q}^{2}\right)^{\epsilon},
$$

where $\Gamma(x)$ is the Euler gamma-function, and

$$
\mathcal{K}_{r}^{(\mathcal{R}) B}\left(\vec{q}_{1}, \vec{q}_{2} ; \vec{q}\right)=\frac{g^{2} c_{R}}{(2 \pi)^{D-1}}\left(\frac{\vec{q}_{1}^{2} \vec{q}_{2}^{\prime 2}+\vec{q}_{2}^{2} \vec{q}_{1}^{\prime 2}}{\left(\vec{q}_{1}-\vec{q}_{2}\right)^{2}}-\vec{q}^{2}\right),
$$

where the superscript $B$ means the leading (Born) approximation and the colour group coefficients $c_{R}$ for the most important cases of the singlet $(R=1)$ and the antisymmetric octet $(R=8)$ representations are $c_{1}=N, c_{8}=\frac{N}{2}$. 
The representation (田) for the scattering amplitudes, as well as the equation (5) for the Green's function and the form (6) of the kernel, are valid in NLA as well as in LLA. Both the kernel of the BFKL equation and the impact factors are unambiguously defined in terms of the gluon Regge trajectory and the effective vertices for the Reggeon-particle interactions [6].

At the two-loop level the gluon trajectory was calculated in [9]. It has both gluon and quark contributions. In the case of $n_{f}$ massless quark flavors it can be presented as

$$
\omega^{(2)}(t)=\frac{g^{2} t}{(2 \pi)^{D-1}} \int \frac{d^{(D-2)} q_{1}}{\vec{q}_{1}^{2} \vec{q}_{1}^{\prime 2}}\left[F\left(\vec{q}_{1}, \vec{q}\right)-2 F\left(\vec{q}_{1}, \vec{q}_{1}\right)\right],
$$

where

$$
\begin{gathered}
F\left(\overrightarrow{q_{1}}, \vec{q}\right)=\frac{2 g^{2} N n_{f} \Gamma\left(2-\frac{D}{2}\right) \Gamma^{2}\left(\frac{D}{2}\right)}{(4 \pi)^{\frac{D}{2}} \Gamma(D)\left(\vec{q}^{2}\right)^{\left(2-\frac{D}{2}\right)}}-\frac{g^{2} N^{2} \vec{q}^{2}}{4(2 \pi)^{D-1}} \int \frac{d^{(D-2)} q_{2}}{\vec{q}_{2}^{2}\left(\vec{q}_{2}-\vec{q}\right)^{2}} \\
\times\left[\ln \left(\frac{\vec{q}^{2}}{\left(\vec{q}_{1}-\vec{q}_{2}\right)^{2}}\right)-2 \psi(D-3)-\psi\left(3-\frac{D}{2}\right)+2 \psi\left(\frac{D}{2}-2\right)+\psi(1)\right. \\
\left.+\frac{2}{(D-3)(D-4)}+\frac{D-2}{4(D-1)(D-3)}\right],
\end{gathered}
$$

$\psi(x)=\Gamma^{\prime}(x) / \Gamma(x)$. Let us stress that we use perturbative expansion in terms of the bare coupling $g$ related to the renormalized coupling $g_{\mu}$ in the $\overline{M S}$ scheme by the relation

$$
g=g_{\mu} \mu^{-\epsilon}\left[1+\left(\frac{11}{3}-\frac{2}{3} \frac{n_{f}}{N}\right) \frac{\bar{g}_{\mu}^{2}}{2 \epsilon}\right], \quad \bar{g}_{\mu}^{2}=\frac{g_{\mu}^{2} N \Gamma(1-\epsilon)}{(4 \pi)^{2+\epsilon}} .
$$

In the limit $\epsilon \rightarrow 0$ we have [9]:

$$
\begin{gathered}
\omega(t)=-\bar{g}_{\mu}^{2}\left(\frac{\vec{q}^{2}}{\mu^{2}}\right)^{\epsilon} \frac{2}{\epsilon}\left\{1+\frac{\bar{g}_{\mu}^{2}}{\epsilon}\left[\left(\frac{11}{3}-\frac{2}{3} \frac{n_{f}}{N}\right)\left(1-\frac{\pi^{2}}{6} \epsilon^{2}\right)-\right.\right. \\
\left(\frac{\vec{q}^{2}}{\mu^{2}}\right)^{\epsilon}\left(\frac{11}{6}+\left(\frac{\pi^{2}}{6}-\frac{67}{18}\right) \epsilon+\left(\frac{202}{27}-\frac{11 \pi^{2}}{18}-\zeta(3)\right) \epsilon^{2}-\right. \\
\left.\left.\left.\frac{n_{f}}{3 N}\left(1-\frac{5}{3} \epsilon+\left(\frac{28}{9}-\frac{\pi^{2}}{3}\right) \epsilon^{2}\right)\right)\right]\right\} .
\end{gathered}
$$

\section{Non-forward BFKL kernel}

The general form of the kernel for arbitrary representation $\mathcal{R}$ of the colour group in the $t$ channel is given by (6). The "virtual" part of the kernel is universal (does not depend on $\mathcal{R}$ ) and is determined by the gluon Regge trajectory. The "real" part can be presented as [6]:

$$
\begin{gathered}
\mathcal{K}_{r}^{(\mathcal{R})}\left(\vec{q}_{1}, \vec{q}_{2} ; \vec{q}\right)=\frac{\left\langle c_{1} c_{1}^{\prime}\left|\hat{\mathcal{P}}_{\mathcal{R}}\right| c_{2} c_{2}^{\prime}\right\rangle}{2 n_{\mathcal{R}}} \int_{0}^{\infty} \frac{d s_{R R}}{(2 \pi)^{D}} \theta\left(s_{\Lambda}-s_{R R}\right) \sum_{\{f\}} \int \gamma_{c_{1} c_{2}}^{\{f\}}\left(q_{1}, q_{2}\right)\left(\gamma_{c_{1}^{\prime} c_{2}^{\prime}}^{\{f\}}\left(q_{1}^{\prime}, q_{2}^{\prime}\right)\right)^{*} d \rho_{f} \\
-\frac{1}{2} \int \frac{d^{D-2} r}{\vec{r}^{2} \vec{r}^{\prime 2}} \mathcal{K}_{r}^{(\mathcal{R}) B}\left(\overrightarrow{q_{1}}, \vec{r} ; \vec{q}\right) \mathcal{K}_{r}^{(\mathcal{R}) B}\left(\vec{r}, \overrightarrow{q_{2}} ; \vec{q}\right) \ln \left(\frac{s_{\Lambda}^{2}}{\left(\vec{r}-\vec{q}_{1}\right)^{2}\left(\vec{r}-\vec{q}_{2}\right)^{2}}\right)
\end{gathered}
$$

where $\hat{\mathcal{P}}_{\mathcal{R}}$ is the operator for projection of two-gluon colour state in the $t$-channel on the representation $R, n_{\mathcal{R}}$ is the number of independent states in $\mathcal{R}, q_{1}=\beta p_{A}+q_{1 \perp}$ and $-q_{2}=$ $\alpha p_{B}-q_{2 \perp}$ are the Reggeon momenta, $s_{R B}=\left(q_{1}-q_{2}\right)^{2}=s \alpha \beta-\left(\vec{q}_{1}-\vec{q}_{2}\right)^{2}$, the sum $\{f\}$ is 
performed over all states $f$ which are produced in the Reggeon-Reggeon collisions and over all discreet quantum numbers of these states, $d \rho_{f}$ is the phase space element for such state,

$$
d \rho_{f}=\frac{1}{n !}(2 \pi)^{D} \delta^{(D)}\left(q_{1}-q_{2}-\sum_{i \in f} k_{i}\right) \prod_{i \in f} \frac{d^{D-1} k_{i}}{(2 \pi)^{D-1} 2 \epsilon_{i}},
$$

$n$ is a number of identical particles in the state $f$. The second term in the r.h.s. of (13) serves for the subtraction of the contribution of the large $s_{R R}$ region in the first term, in order to avoid a double counting of this region in the BFKL equation. The intermediate parameter $s_{\Lambda}$ in (13) must be taken tending to infinity. In LLA only the one-gluon production does contribute and (13) gives for the kernel its LLA value (8); in NLA the contributing states include also two-gluon and quark-antiquark states. At large $s_{R R}$ only the contribution of the two-gluon production does survive in the first integral, so that the dependence on $s_{\Lambda}$ disappears in (13) due to the factorization property of the two-gluon production vertex [6].

The remarkable properties of the kernel are

$$
\mathcal{K}_{r}^{(\mathcal{R})}\left(0, \vec{q}_{2} ; \vec{q}\right)=\mathcal{K}_{r}^{(\mathcal{R})}\left(\vec{q}_{1}, 0 ; \vec{q}\right)=\mathcal{K}_{r}^{(\mathcal{R})}\left(\vec{q}, \vec{q}_{2} ; \vec{q}\right)=\mathcal{K}_{r}^{(\mathcal{R})}\left(\overrightarrow{q_{1}}, \vec{q} ; \vec{q}\right)=0
$$

and

$$
\mathcal{K}_{r}^{(\mathcal{R})}\left(\vec{q}_{1}, \vec{q}_{2} ; \vec{q}\right)=\mathcal{K}_{r}^{(\mathcal{R})}\left(\vec{q}_{1}^{\prime}, \vec{q}_{2}^{\prime} ;-\vec{q}\right)=\mathcal{K}_{r}^{(\mathcal{R})}\left(-\vec{q}_{2},-\vec{q}_{1} ;-\vec{q}\right) .
$$

The properties (15) mean that the kernel turns into zero at zero transverse momenta of the Reggeons and follow from the gauge invariance; (16) are the consequences of the symmetry of the r.h.s. of $(13)$.

The most interesting representations $\mathcal{R}$ are the colour singlet (Pomeron channel) and the antisymmetric colour octet (gluon channel). We have for the singlet case

$$
\left\langle c_{1} c_{1}^{\prime}\left|\hat{\mathcal{P}}_{1}\right| c_{2} c_{2}^{\prime}\right\rangle=\frac{\delta_{c_{1} c_{1}^{\prime}} \delta_{c_{2} c_{2}^{\prime}}}{N^{2}-1}, \quad n_{1}=1
$$

and for the antisymmetric octet case

$$
\left\langle c_{1} c_{1}^{\prime}\left|\hat{\mathcal{P}}_{8-}\right| c_{2} c_{2}^{\prime}\right\rangle=\frac{f_{c_{1} c_{1}^{\prime} c} f_{c_{2} c_{2}^{\prime} c}}{N}, \quad n_{8}=N^{2}-1
$$

where $f_{a b c}$ are the structure constants of the colour group.

The quark contribution to the "real" part for any representation $\mathcal{R}$ is given by a superposition of the Abelian and non-Abelian pieces [10]. The Abelian piece was calculated many yes ago in the framework of Quantum Electrodynamics [11], but, unfortunately, it has rather complicated form and can not be presented here. In the gluon channel this piece is absent and the quark contribution in this channel has very simple form:

$$
\begin{gathered}
\mathcal{K} r^{(8-) Q}\left(\vec{q}_{1}, \vec{q}_{2} ; \vec{q}\right)=\frac{g^{4} n_{f} N}{(2 \pi)^{D-1}} \frac{\Gamma(1-\epsilon)}{\epsilon(4 \pi)^{2+\epsilon}} \frac{\left.\Gamma^{2}(2+\epsilon)\right]}{\Gamma(4+2 \epsilon)}\left\{\frac{\left(\vec{k}^{2}\right)^{\epsilon}}{\vec{k}^{2}}\left(\vec{q}_{1}^{2} \vec{q}_{2}^{\prime 2}+\vec{q}_{2}^{2} \vec{q}_{1}^{\prime 2}\right)+\vec{q}^{2}\left(\left(\vec{q}^{2}\right)^{\epsilon}\right.\right. \\
\left.\left.-\left(\vec{q}_{1}^{2}\right)^{\epsilon}-\left(\vec{q}_{2}^{2}\right)^{\epsilon}\right)-\frac{\left(\vec{q}_{1}^{2} \vec{q}_{2}^{\prime 2}-\vec{q}_{2}^{2} \vec{q}_{1}^{\prime 2}\right)}{\vec{k}^{2}}\left(\left(\vec{q}_{1}^{2}\right)^{\epsilon}-\left(\vec{q}_{2}^{2}\right)^{\epsilon}\right)+\left(\vec{q}_{1} \leftrightarrow \vec{q}_{1}^{\prime}, \vec{q}_{2} \leftrightarrow \vec{q}_{2}^{\prime}\right)\right\},
\end{gathered}
$$

where $\vec{k}=\vec{q}_{1}-\vec{q}_{2}=\vec{q}_{1}^{\prime}-\vec{q}_{2}^{\prime}$. The properties (15), (16) are evident from (19).

The gluon piece of the "real" part of the kernel includes contributions of the one-gluon and two-gluon productions. The one-gluon contribution is determined by the RRG-vertex and depends on the representation $\mathcal{R}$ only through the numerical coefficient $c_{R}$ in the same way 
as in the Born case (8). The calculation of the RRG-vertex has a long history. The quark part of the vertex was calculated at once at arbitrary $D$ [12. Instead, in the gluon part firstly only the terms finite at $\epsilon \rightarrow 0$ were kept [13]. But then it was understood that in order to find the BFKL kernel the RRG-vertex must be calculated at arbitrary $D$ in the region of small transverse momenta $\vec{k}$ of the produced gluon. It was done in $\llbracket 14$. Later the results of [13, 14] were obtained by another method in [15]. But after all it appeared [16] that for the verification of the "bootstrap" equation the vertex must be known at arbitrary $D$ in a wider kinematical region. Therefore it became clear that the vertex should be calculated at arbitrary $D$, especially taking into account that it can be used not only for the check of the "bootstrap", but, for example, in the Odderon problem at NLO and so on. Quite recently [17] the vertex and corresponding contribution to the non-forward BFKL kernel were calculated at arbitrary $D$.

The two-gluon contribution, analogously to the quark one, for any representation $\mathcal{R}$ is given by a linear combination of two pieces. Only one of them enters in the antisymmetric octet kernel. It was calculated recently [18]. In the case of arbitrary $D$ the integral representation was obtained.

We have not enough space here to show neither the one-gluon nor the two-gluon contributions at arbitrary $D$, so that we present only the sum of these contributions to the "real" part of the BFKL kernel for the antisymmetric octet representation in the limit $\epsilon \rightarrow 0$ [18]:

$$
\begin{gathered}
\mathcal{K}_{r}^{G(8-)}\left(\vec{q}_{1}, \vec{q}_{2} ; \vec{q}\right)=\frac{g^{2} N}{2(2 \pi)^{D-1}}\left\{\left(\frac{\vec{q}_{1}^{2} \vec{q}_{2}^{\prime 2}+\vec{q}_{1}^{\prime 2} \vec{q}_{2}^{2}}{\vec{k}^{2}}-\vec{q}^{2}\right)\right. \\
\times\left(1+\frac{g^{2} N \Gamma(1-\epsilon)\left(\vec{k}^{2}\right)^{\epsilon}}{(4 \pi)^{2+\epsilon}}\left(-\frac{11}{6 \epsilon}+\frac{67}{18}-\zeta(2)+\epsilon\left(-\frac{202}{27}+7 \zeta(3)+\frac{11}{6} \zeta(2)\right)\right)\right) \\
+\frac{g^{2} N \Gamma(1-\epsilon)}{(4 \pi)^{2+\epsilon}}\left[\vec{q}^{2}\left(\frac{11}{6} \ln \left(\frac{\vec{q}_{1}^{2} \vec{q}_{2}^{2}}{\vec{q}^{2} \vec{k}^{2}}\right)+\frac{1}{4} \ln \left(\frac{\vec{q}_{1}^{2}}{\vec{q}^{2}}\right) \ln \left(\frac{\vec{q}_{1}^{\prime 2}}{\vec{q}^{2}}\right)+\frac{1}{4} \ln \left(\frac{\vec{q}_{2}^{2}}{\vec{q}^{2}}\right) \ln \left(\frac{\vec{q}_{2}^{\prime 2}}{\vec{q}^{2}}\right)\right)\right. \\
+\frac{1}{4} \ln ^{2}\left(\frac{\vec{q}_{1}^{2}}{\vec{q}_{2}^{2}}\right)-\frac{\vec{q}_{1}^{2} \vec{q}_{2}^{\prime 2}+\vec{q}_{2}^{2} \vec{q}_{1}^{2}}{2 \vec{k}^{2}} \ln ^{2}\left(\frac{\vec{q}_{1}^{2}}{\vec{q}_{2}^{2}}\right)+\frac{\vec{q}_{1}^{2} \vec{q}_{2}^{\prime 2}-\vec{q}_{2}^{2} \vec{q}_{1}^{\prime 2}}{\vec{k}^{2}} \ln \left(\frac{\vec{q}_{1}^{2}}{\vec{q}_{2}^{2}}\right)\left(\frac{11}{6}-\frac{1}{4} \ln \left(\frac{\vec{q}_{1}^{2} \vec{q}_{2}^{2}}{\vec{k}^{4}}\right)\right) \\
+\frac{1}{2}\left[\vec{q}^{2}\left(\vec{k}^{2}-\vec{q}_{1}^{2}-\vec{q}_{2}^{2}\right)+2 \vec{q}_{1}^{2} \vec{q}_{2}^{2}-\vec{q}_{1}^{2} \vec{q}_{2}^{\prime 2}-\vec{q}_{2}^{2} \vec{q}_{1}^{2}+\frac{\vec{q}_{1}^{2} \vec{q}_{2}^{\prime 2}-\vec{q}_{2}^{2} \vec{q}_{1}^{\prime 2}}{\vec{k}^{2}}\left(\vec{q}_{1}^{2}-\vec{q}_{2}^{2}\right)\right] \\
\left.\left.\times \int_{0}^{1} \frac{g^{2} N}{\left(\vec{q}_{1}(1-x)+\vec{q}_{2} x\right)^{2}} \ln \left(\frac{\vec{q}_{1}^{2}(1-x)+\vec{q}_{2}^{2} x}{\vec{k}^{2} x(1-x)}\right)\right]\right\}+\frac{\left.\vec{q}_{i} \longleftrightarrow \vec{q}_{i}^{\prime}\right\},}{2(2 \pi)^{D-1}} \longleftrightarrow
\end{gathered}
$$

where $\zeta(n)$ is the Riemann zeta-function. Note, that the the one-gluon and the two-gluon contributions separately have singularities $\sim 1 / \epsilon^{2}$. Due to their cancellation the leading singularity of the kernel is $1 / \epsilon$. It turns again into $\sim 1 / \epsilon^{2}$ after subsequent integrations of the kernel because of the singular behaviour of the kernel at $\vec{k}^{2}=0$. The additional singularity arises from the region of small $\vec{k}^{2}$, where $\epsilon\left|\ln \vec{k}^{2}\right| \sim 1$. Therefore we have not expanded in $\epsilon$ the term $\left(\vec{k}^{2}\right)^{\epsilon}$. The terms $\sim \epsilon$ are taken into account in the coefficient of the divergent at $\vec{k}^{2}=0$ expression in order to conserve all giving nonvanishing in the limit $\epsilon \rightarrow 0$ contributions after the integrations.

The integral in (20) can be presented in another form:

$$
\int_{0}^{1} \frac{d x}{\left(\vec{q}_{1}(1-x)+\vec{q}_{2} x\right)^{2}} \ln \left(\frac{\vec{q}_{1}^{2}(1-x)+\vec{q}_{2}^{2} x}{\vec{k}^{2} x(1-x)}\right)=\int_{0}^{\infty} \frac{d z}{z+\vec{k}^{2}} \frac{1}{\sqrt{\left(\vec{q}_{1}^{2}+\vec{q}_{2}^{2}+z\right)^{2}-4 \vec{q}_{1}^{2} \vec{q}_{2}^{2}}}
$$




$$
\times \ln \left(\frac{\vec{q}_{1}^{2}+\vec{q}_{2}^{2}+z+\sqrt{\left(\vec{q}_{1}^{2}+\vec{q}_{2}^{2}+z\right)^{2}-4 \vec{q}_{1}^{2} \vec{q}_{2}^{2}}}{\vec{q}_{1}^{2}+\vec{q}_{2}^{2}+z-\sqrt{\left(\vec{q}_{1}^{2}+\vec{q}_{2}^{2}+z\right)^{2}-4 \vec{q}_{1}^{2} \vec{q}_{2}^{2}}}\right) .
$$

It is possible also to express the integral in (20) in terms of dilogarithms, but this expression is not very convenient:

$$
\begin{gathered}
\int_{0}^{1} \frac{d x}{\left(\vec{q}_{1}(1-x)+\vec{q}_{2} x\right)^{2}} \ln \left(\frac{\vec{q}_{1}^{2}(1-x)+\vec{q}_{2}^{2} x}{\vec{k}^{2} x(1-x)}\right)=-\frac{2}{\left|\vec{q}_{1}\right|\left|\vec{q}_{2}\right| \sin \phi} \\
\times\left[\ln \rho \arctan \frac{\rho \sin \phi}{(1-\rho \cos \phi)}+\operatorname{Im}(L(\rho \exp i \phi))\right],
\end{gathered}
$$

where $\phi$ is the angle between $\vec{q}_{1}$ and $\vec{q}_{2}$,

$$
\rho=\min \left(\frac{\left|\vec{q}_{1}\right|}{\left|\vec{q}_{2}\right|}, \frac{\left|\vec{q}_{2}\right|}{\left|\vec{q}_{1}\right|}\right), L(z)=\int_{0}^{z} \frac{d t}{t} \ln (1-t) .
$$

The symmetries (16) of the kernel are easily seen. The first of them is explicit in (20). To notice the second it is sufficient to change $x \leftrightarrow(1-x)$ in the integral in (20).

In order to check that the kernel (20) turns into zero at zero transverse momenta of Reggeons one has to know the behaviour of the integral in (20). A suitable for this purpose representation is given in (21). From this representation one can see that singularities of the integral at zero transverse momenta of the Reggeons are not more than logarithmic. After this no problems remain to verify (15).

\section{Non-forward impact factors}

The impact factors can be expressed through the effective vertices $\Gamma_{\{(f)\} P}^{c}$. In the NLLA the representation takes the form [6]:

$$
\begin{aligned}
& \Phi_{P^{\prime} P}^{(\mathcal{R}, \nu)}\left(\vec{q}_{R} ; \vec{q} ; s_{0}\right)=\left\langle c c^{\prime}\left|\hat{\mathcal{P}}_{\mathcal{R}}\right| \nu\right\rangle \int \frac{d s_{P R}}{2 \pi} \theta\left(s_{\Lambda}-s_{P R}\right) \sum_{\{f\}} \int \Gamma_{\{f\} P}^{c}\left(q_{R}\right)\left(\Gamma_{\{f\} P^{\prime}}^{c^{\prime}}\left(q_{R}^{\prime}\right)\right)^{*}\left(\frac{s_{0}}{{\overrightarrow{q_{R}}}^{2}}\right)^{\frac{1}{2} \omega\left(-\vec{q}_{R}^{2}\right)} \\
& \quad \times\left(\frac{s_{0}}{\vec{q}_{R}^{\prime 2}}\right)^{\frac{1}{2} \omega\left(-\vec{q}_{R}^{\prime 2}\right)} d \rho_{f}-\frac{1}{2} \int \frac{d^{D-2} r}{\vec{r}^{2} \vec{r}^{\prime 2}} \Phi_{P^{\prime} P}^{(\mathcal{R}, \nu) B}(\vec{r}, \vec{q}) \mathcal{K}_{r}^{(\mathcal{R}) B}\left(\vec{r}, \vec{q}_{R} ; \vec{q}\right) \ln \left(\frac{s_{\Lambda}^{2}}{\left(\vec{r}-\vec{q}_{R}\right)^{2} s_{0}}\right) \cdot \quad(24)
\end{aligned}
$$

Here $s_{P R}=\left(p_{P}-q_{R}\right)^{2}$ is the squared particle-Reggeon invariant mass. The argument $s_{0}$ in the impact factor shows that it depends on the energy scale $s_{0}$ of the Mellin transformation in (स1). Of course, this dependence disappears in the total expression for the amplitudes (4). The Born (LLA) impact factors $\Phi_{P^{\prime} P}^{(\mathcal{R}, \nu) B}$ are given by the first term in the r.h.s. of (24) taken in the Born approximation. Note that for the Born case the integral over $s_{P R}$ in (24) is convergent, so that the parameter $s_{\Lambda}$ does not play any role. In NLA the independence of the impact factors on $s_{\Lambda}$ is guaranteed by the factorization properties of the effective vertices $\Gamma_{\{(f)\} P}^{c}[6]$.

The non-forward impact factors of gluon and quark were calculated at NLO in [19 and 20] correspondingly for arbitrary $\mathcal{R}$. Their general form is rather complicated, so that we present here these impact factors only for the antisymmetric octet representation (we omit the superscript $\mathcal{R}=8-$, since we consider below only this representation):

$$
\Phi_{G^{\prime} G}^{a}\left(\vec{q}_{1}, \vec{q}, s_{0}\right)=\frac{-i g^{2} \sqrt{N}}{2} T_{G^{\prime} G}^{a}\left[\delta _ { \lambda _ { G } , \lambda _ { G ^ { \prime } } } \left\{1+\frac{\omega^{(1)}(t)}{2}\left[\tilde{K}_{1}+\left(\left(\frac{\vec{q}_{1}^{2}}{\vec{q}^{2}}\right)^{\epsilon}+\left(\frac{\vec{q}_{1}^{2}}{\vec{q}^{2}}\right)^{\epsilon}-1\right)\right.\right.\right.
$$




$$
\begin{gathered}
\times\left(\frac{1}{2 \epsilon}+\psi(1+2 \epsilon)-\psi(1+\epsilon)+\frac{11+7 \epsilon}{2(1+2 \epsilon)(3+2 \epsilon)}-\frac{n_{f}}{N} \frac{(1+\epsilon)}{(1+2 \epsilon)(3+2 \epsilon)}\right) \\
+\ln \left(\frac{s_{0}}{\vec{q}^{2}}\right)+\frac{3}{2 \epsilon}+2 \psi(1)-\psi(1+\epsilon)-\psi(1+2 \epsilon) \\
\left.\left.-\frac{9(1+\epsilon)^{2}+2}{2(1+\epsilon)(1+2 \epsilon)(3+2 \epsilon)}+\frac{n_{f}}{N} \frac{(1+\epsilon)^{3}+\epsilon^{2}}{(1+\epsilon)^{2}(1+2 \epsilon)(3+2 \epsilon)}\right]\right\} \\
\left.-\frac{\delta_{\lambda_{G},-\lambda_{G^{\prime}}} \epsilon \omega^{(1)}(t)}{2(1+\epsilon)^{2}(1+2 \epsilon)(3+2 \epsilon)}\left(1+\epsilon-\frac{n_{f}}{N}\right)\right] \\
\Phi_{Q^{\prime} Q}^{a}\left(\vec{q}_{1}, \vec{q}, s_{0}\right)=\frac{-i g^{2} \sqrt{N}}{2} T_{Q^{\prime} Q}^{a} \delta_{\lambda_{Q}, \lambda_{Q^{\prime}}}\left(1+\frac{\omega^{(1)}(t)}{2}\left[\tilde{K}_{1}+\left(\left(\frac{\vec{q}_{1}^{2}}{\vec{q}^{2}}\right)^{\epsilon}+\left(\frac{\vec{q}_{1}^{\prime 2}}{\vec{q}^{2}}\right){ }^{\epsilon}\right)\left\{\frac{1}{2 \epsilon}\right.\right.\right. \\
\left.+\psi(1+2 \epsilon)-\psi(1+\epsilon)+\frac{11+7 \epsilon}{2(1+2 \epsilon)(3+2 \epsilon)}-\frac{n_{f}}{N} \frac{(1+2 \epsilon)(3+2 \epsilon)}{(1+2)}\right\} \\
\left.\left.+\ln \left(\frac{s_{0}}{\vec{q}^{2}}\right)+2 \psi(1)-2 \psi(1+2 \epsilon)-\frac{1}{2(1+2 \epsilon)}-\frac{1}{N^{2}}\left(\frac{(3-2 \epsilon)}{\epsilon(1+2 \epsilon)}\right)\right]\right)
\end{gathered}
$$

where $T_{P^{\prime} P}^{a}$ are the matrix elements of the colour group generators in corresponding representations, $\lambda_{P}$ are the particle helicities and the function $\tilde{K}_{1}$ has the following integral representation:

$$
\tilde{K}_{1}=\frac{(4 \pi)^{2+\epsilon} \Gamma(1+2 \epsilon) \epsilon\left(\vec{q}^{2}\right)^{-\epsilon}}{4 \Gamma(1-\epsilon) \Gamma^{2}(1+\epsilon)} \int \frac{d^{D-2} k}{(2 \pi)^{D-1}} \ln \left(\frac{\vec{q}^{2}}{\vec{k}^{2}}\right) \frac{\vec{q}^{2}}{\left(\vec{k}-\vec{q}_{1}\right)^{2}\left(\vec{k}-\vec{q}_{1}^{\prime}\right)^{2}} .
$$

The result of the integration of (27), in form of expansion in $\epsilon$, can be found in [19].

Of course, the most interesting is the impact factor for virtual photon, since it is the impact factor of physical particle which can be calculated in perturbative QCD. In the leading order it differs only by a numerical factor from the QED case, which is known long ago. Unfortunately, at NLO this impact factor is not yet calculated, although some steps in this direction are done [21], [22].

\section{5 "Bootstrap" of the gluon Reggeization}

The "bootstrap" condition for the kernel derived in Ref. [6] has the form

$$
\int \frac{d^{D-2} q_{1}}{\vec{q}_{1}^{2} \vec{q}_{1}^{\prime 2}}\left[\int \frac{d^{D-2} q_{2}}{\vec{q}_{2}^{2} \vec{q}_{2}^{\prime 2}} \mathcal{K}^{(8-)}\left(\vec{q}_{1}, \overrightarrow{q_{2}} ; \vec{q}\right)-\omega(t)\right]=0
$$

where $\mathcal{K}^{(8-)}\left(\vec{q}_{1}, \vec{q}_{2} ; \vec{q}\right)$ is the kernel of the non-forward BFKL equation in the gluon (antisymmetric octet) channel, $\omega(t)=\omega^{(1)}(t)+\omega^{(2)}(t)$ is the deviation of the gluon Regge trajectory from unity and $t=-\vec{q}^{2}$.

The fulfillment of this condition for the quark contribution to the kernel was shown in [10] at arbitrary $D$. In the one-loop approximation the trajectory is purely gluonic (see (77)). The quark contribution to the trajectory appears at the two-loop level and is given by the terms proportional to $n_{f}$ in $(9)$, (10). The kernel $\mathcal{K}^{(8)}\left(\vec{q}_{1}, \vec{q}_{2} ; \vec{q}\right)$, according to (6), is expressed through the trajectory and the "real" part. The quark piece of the latter is given by (19). Using this equation together with (9), (10) for the quark contribution to the trajectory, we obtain

$$
\int \frac{d^{D-2} q_{2}}{\vec{q}_{2}^{2} \vec{q}_{2}^{\prime 2}} \mathcal{K}^{(8-) Q}\left(\vec{q}_{1}, \vec{q}_{2} ; \vec{q}\right)=g^{4} n_{f} N \frac{1}{(2 \pi)^{D-1}} \frac{1}{(4 \pi)^{2+\epsilon}} \frac{\Gamma(1-\epsilon)}{\epsilon} \frac{[\Gamma(2+\epsilon)]^{2}}{\Gamma(4+2 \epsilon)}
$$




$$
\begin{gathered}
\times \int \frac{d^{D-2} q_{2}}{\vec{q}_{2}^{2}}\left\{\frac{\vec{q}_{1}^{2}}{\vec{k}^{2}}\left(\left(\vec{q}_{1}^{2}\right)^{\epsilon}+\left(\vec{q}_{1}^{\prime 2}\right)^{\epsilon}-\left(\vec{q}_{2}^{2}\right)^{\epsilon}-\left(\vec{q}_{2}^{\prime 2}\right)^{\epsilon}\right)\right. \\
\left.+\frac{\vec{q}^{2}}{{\overrightarrow{q_{2}^{\prime}}}^{2}}\left(\left(\vec{q}^{2}\right)^{\epsilon}-\left(\vec{q}_{1}^{2}\right)^{\epsilon}-\left(\vec{q}_{2}^{2}\right)^{\epsilon}\right)+\left(\vec{q}_{1} \longleftrightarrow \vec{q}_{1}^{\prime}\right)\right\},
\end{gathered}
$$

where $\vec{k}=\vec{q}_{1}-\vec{q}_{2}, \vec{q}_{i}^{\prime}=\vec{q}_{i}-\vec{q}$. Putting (29) in (28) and using (9), (10), it is easy to verify that the "bootstrap" equation (28) is satisfied.

To demonstrate the fulfillment of the "bootstrap" condition for the gluon piece of the kernel is not so simple. In [16] it was done for the terms in the gluon trajectory non-vanishing for $\epsilon \rightarrow 0$. Even for this purpose the knowledge of the "real" part of the kernel $\mathcal{K}_{r}^{G(8-)}\left(\overrightarrow{q_{1}}, \overrightarrow{q_{2}} ; \vec{q}\right)$ (20) is not sufficient on account of the singularity of the integration measure in (28) at zero momenta of scattered Reggeons. Fortunately, $\mathcal{K}_{r}^{G\left({ }^{-}-\right)}\left(\vec{q}_{1}, \vec{q}_{2} ; \vec{q}\right)$ turns into zero at this points (see (15)), so that at first sight these points could not bring additional singularities in $\epsilon$. It would be so if integration over only one momentum was performed. But in the region where the momenta of two Reggeons (let us say, $\overrightarrow{q_{1}}$ and $\overrightarrow{q_{2}}$ ) turn into zero simultaneously, being of the same order, $\mathcal{K}_{r}^{G\left({ }^{(8-}\right)}\left(\vec{q}_{1}, \overrightarrow{q_{2}} ; \vec{q}\right)$ does not vanish (as it can be easily seen in the example of the Born case (8)). Therefore, these regions can give additional singularities in $\epsilon$; moreover, since integration over these regions leads to singularities, an expansion of, let us say, $\left(\vec{q}_{1}^{2}\right)^{\epsilon}$ and $\left(\vec{q}_{2}^{2}\right)^{\epsilon}$ is not possible anymore, so that we need to know about the kernel more than it is given by (24). This problem was solved in [16] and the fulfillment of the "bootstrap" equation (28) was proved for the terms in $\omega^{(2)}$ non-vanishing for $\epsilon \rightarrow 0$. After the calculation of the RRG vertex at arbitrary $D$ [17] examination of the "bootstrap" condition became possible at any you like dimension. This question is under consideration now.

The "bootstrap" conditions for the impact factors derived in [6] are:

$$
\begin{gathered}
i g \frac{\sqrt{N} t}{(2 \pi)^{D-1}} \int \frac{d^{D-2} q_{1}}{{\overrightarrow{q_{1}}}^{2}{\vec{q}_{1}^{\prime}}^{2}} \Phi_{P^{\prime} P}^{c}\left(\vec{q}_{1}^{\prime} ; \vec{q} ; s_{0}\right) \\
=\Gamma_{P^{\prime} P}^{c}(q) \omega^{(1)}(t)+\Gamma_{P^{\prime} P}^{c(B)}(q) \frac{1}{2}\left[\omega^{(2)}(t)-\left(\omega^{(1)}(t)\right)^{2} \ln \left(\frac{\vec{q}^{2}}{s_{0}}\right)\right] .
\end{gathered}
$$

The fulfillment of these conditions for the gluon and quark impact factors was demonstrated in [19] and [20] correspondingly at arbitrary $D$.

More strong conditions than (28), (30) were suggested in [23] (from another point of view they were considered in [24]). This suggestion is based on the requirement of Reggeization of the unphysical particle-Reggeon scattering amplitude with colour octet in the $t$-channel and negative signature. It was shown in [23], [24] that the strong bootstrap conditions are satisfied in the case of the gluon and quark impact factors. It is rather intriguing, because their role is not yet clear.

\section{References}

[1] V.S. Fadin, E.A. Kuraev and L.N. Lipatov, Phys. Lett. B 60 (1975) 50; E.A. Kuraev, L.N. Lipatov and V.S. Fadin, Zh. Eksp. Teor. Fiz. 71 (1976) 840; 72 (1977) 377; Ya. Ya. Balitskii and L.N. Lipatov, Sov. J. Nucl. Phys. 28 (1978) 822.

[2] V.S. Fadin and L.N. Lipatov, Phys. Lett. B 429 (1998) 127.

[3] M. Ciafaloni and G. Camici, Phys. Lett. B 430 (1998) 349. 
[4] S.J. Brodsky, V.S. Fadin, V.T. Kim, L.N. Lipatov, and G.B. Pivovarov, Pis'ma v ZhETF, 70 (1999) 161; JETP Lett. 70 (1999) 155.

[5] D.A. Ross, Phys. Lett. B431 (1998) 161; Yu.V. Kovchegov and A.H. Mueller, Phys. Lett. B439 (1998) 423; J. Blümlein, V. Ravindran, W.L. van Neerven and A. Vogt, preprint DESY-98-036, hep-ph/9806368; E.M. Levin, preprint TAUP 2501-98, hep-ph/9806228; N. Armesto, J. Bartels, M.A. Braun, Phys. Lett. B442 (1998) 459; G.P. Salam, JHEP 8907 (1998) 19; M. Ciafaloni and D. Colferai, Phys. Lett. B452 (1999) 372; M. Ciafaloni, D. Colferai and G.P. Salam, Phys. Rev. D60 (1999) 114036; R.S. Thorne, Phys. Rev. D60 (1999) 054031.

[6] V.S. Fadin and R. Fiore, Phys. Lett. B 440 (1998) 359.

[7] V.S. Fadin, V.E. Sherman, ZhETF Pis'ma 23 (1976) 599; ZhETF 72 (1977) 1640.

[8] V.S. Fadin, hep-ph/9807528; Proceedings of LAFEX International School on High Energy Physics (LISHEP 98) Session C, Rio de Janeiro, Brazil, Ed. A. Brandt, H. Motta, A. Santoro, pp. 742-776; hep-ph/9807528.

[9] V.S. Fadin, Zh. Eksp. Teor. Fiz. Pis'ma 61 (1995) 342; V.S. Fadin, R. Fiore and A. Quartarolo, Phys. Rev. D 53 (1996) 2729; M.I. Kotsky and V.S. Fadin, Yad. Fiz. 59(6) (1996) 1; V.S. Fadin, R. Fiore and M.I. Kotsky, Phys. Lett. B359 (1995) 181; Phys. Lett. B387 (1996) 593.

[10] V.S. Fadin, R. Fiore and A. Papa, Phys. Rev. D 60, (1999) 074025.

[11] H. Cheng, T.T. Wu, Phys. Rev. D 10 (1970) 2775.

[12] V.S. Fadin, R. Fiore and A. Quartarolo, Phys. Rev. D50 (1994) 5893.

[13] V.S. Fadin, L.N. Lipatov, Nucl. Phys. B406 (1993) 259.

[14] V.S. Fadin, R. Fiore and M.I. Kotsky, Phys. Lett. B389 (1996) 737.

[15] V. Del Duca, C.R. Schmidt, Phys. Rev. D59 (1999) 074004.

[16] V.S. Fadin, R. Fiore and M.I. Kotsky, hep-ph/0007312; Phys.Lett.B494 (2000) 100.

[17] V.S. Fadin, R. Fiore and A. Papa, Budker INP 2000-63; hep-ph/0008006.

[18] V.S. Fadin and D.A. Gorbachev, JETP Letters, 71, 222 (2000); Pis'ma v ZhETF, 71, 322 (2000); Yad. Fiz. 63, N 12 (2000) 1-17.

[19] V.S. Fadin, R. Fiore, M.I. Kotsky and A. Papa, Phys. Rev. D61 (2000) 094005.

[20] V.S. Fadin, R. Fiore, M.I. Kotsky and A. Papa, Phys. Rev. D61 (2000) 094006.

[21] V.S Fadin and A.D. Martin, Phys. Rev. D60 (1999) 114008; V. Fadin, D. Ivanov and M. Kotsky, hep-ph/0007119; New Trends in High Energy Physics; Kiev,2000, Ed. L.L. Jenkovsky, p.190.

[22] J. Bartels, S. Gieseke and C.F. Qiao, hep-ph/0009102; DESY-00-132.

[23] M. Braun, hep-ph/9901447; M. Braun and G.P. Vacca, Phys. Lett. B447 (2000) 156.

[24] V.S. Fadin, R. Fiore, M.I. Kotsky and A. Papa, ]hep-ph/0008057; Phys. Lett. B495 (2000) 329. 